\title{
Complex dielectric response of the CDW ground state in o-TaS 3
}

\author{
D. Staresinic, K. Biljakovic, W. Bruetting*, K. Hosseini* and S. Zaitsev-Zotov** \\ Institute of Physics, P.O. Box 304, 10001 Zagreb, Croatia \\ *Physikal Institut, Uni. Bayreuth, 95440 Bayreuth, Germany \\ ** Institute of Radioengineering and Electronics, Russian Academy of Sciences, Mokhovaya 11, \\ 103907 Moscow, Russia
}

\begin{abstract}
We report the temperature evolution of the low-frequency $(f<10 \mathrm{MHz})$ dielectric response of the CDW material orthorombic $\mathrm{TaS}_{3}$ between $150 \mathrm{~K}$ and $10 \mathrm{~K}$. There are two distinctive processes observed bellow and above $50 \mathrm{~K}$ that define different ground states of $C D W$. The first one due to the weakly pinned elastic $C D W$ freezes at about $50 K$ and a second one due to the dynamics of topological defects of frozen CDW appears below. Similar change of regime is observed in linear conductivity in the same temperature range This second process we detect also in thermally stimulated discharge, an alternative technique of low frequency dielectric measurement, which allows access to effective frequencies down to $0.1 \mathrm{mHz}$.
\end{abstract}

\section{Introduction}

The behavior of CDW due to the interaction with random pinning centers has generally been referred to as "glassy". It seems, yet, that the high temperature properties in the so called weak, or collective, pinning regime and low temperature properties in the so called strong, or local, pinning regime are quite different in many aspects, e.g. the change of the activation energy in the temperature dependence of linear conductivity [1], change of nonlinear regime [2], different processes observed in dielectric relaxation [3]. We performed a detailed measurements of the low frequency dielectric response of standard CDW system, orthorombic $\mathrm{TaS}_{3}$, in wide temperature range from $150 \mathrm{~K}$ down to $10 \mathrm{~K}$ for frequencies below 10 $\mathrm{MHz}$ down to $0.01 \mathrm{~Hz}$, in hope to acquire a better understanding of possible different "glassy" phases of CDW. In addition, the thermally stimulated discharge current measurements (TSDC), as an alternative method to study dielectric properties [4], have been performed at temperatures below $20 \mathrm{~K}$.

\section{Results and discussion}

The experimental conditions for low frequency dielectric spectroscopy [5] and TSDC [6] measurements are described elsewhere, and we present here only main results. In figure 1 . the linear conductivity $\sigma$ and the real part of the dielectric constant $\varepsilon$ measured on the same sample are shown. It is obvious that there is

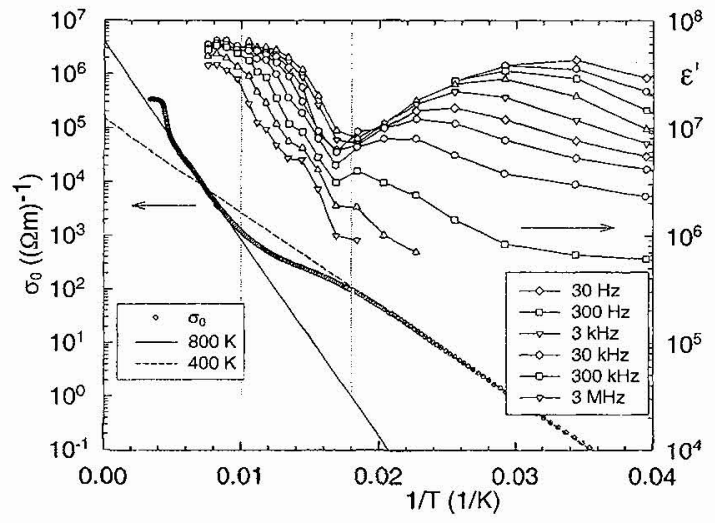

Figure 1. Temperature dependence of conductivity and real part of dielectric constant showing two different regimes above and below $50 \mathrm{~K}$.

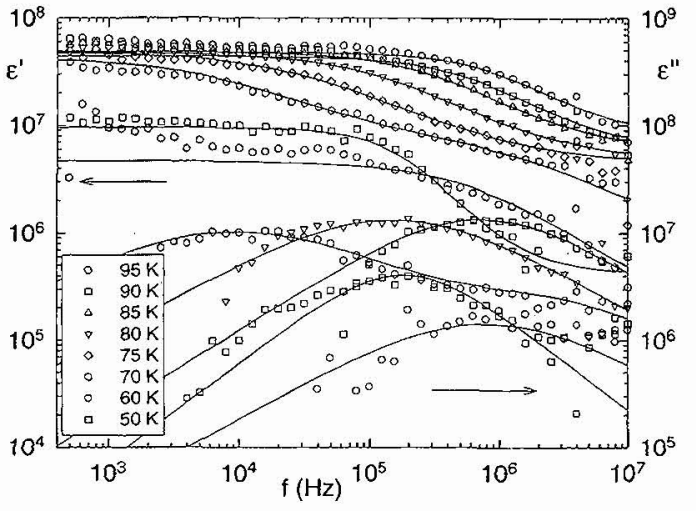

Figure 2. Frequency dependence of real and imaginary part of dielectric constant. Full lines are fits to Cole-Cole function of overdamped response with variable width. 
a crossover regime in the temperature range $50-100 \mathrm{~K}$ where the activation energy of $\sigma$ changes from $800 \mathrm{~K}$ to $400 \mathrm{~K}$ and the dielectric response of CDW decreases substantially down to a well defined minimum after which it increases again. In figure 2 . we present the frequency dependence of real and imaginary part of $\varepsilon$ between $100 \mathrm{~K}$ and $50 \mathrm{~K}$. The characteristic frequency $\omega_{0}$ decreases with temperature leading to the disappearance of this process (we call it $\alpha$ ) from frequency window and a new, $\beta$ process at higher frequencies can be observed. At lower temperatures the amplitude of $\beta$ process increases and $\omega_{0}$ decreases. We present in figure 3. the temperature dependence of the $\omega_{0}$ for both processes. $\alpha$ process decreases faster than the activation law leading to a critical slowing down at about $50 \mathrm{~K}$ as obtained from the Vogel-Fulcher fit [5]. $\beta$ process follows the activation law with the energy of $400 \mathrm{~K}$. As we have not been able to follow $\beta$ process below $20 \mathrm{~K}$, we applied TSDC technique especially suitable for isolators that $\mathrm{CDW}$ systems at low temperatures are. In figure 4 . the current spectra for different polarizing voltages are shown. For low enough voltages there is a well defined peak around $16 \mathrm{~K}$. We measured the change of the peak position, $\mathrm{T}_{\mathrm{M}}$, for various heating rates $h$. For a given $h$ and $\mathrm{T}_{\mathrm{M}}$ the effective frequency can be calculated $[5,7]$ and it should be the same as the characteristic frequency of the dielectric response at $T_{M}$. The inset shows that it continues the temperature dependence obtained for $\beta$ process. TSDC measurements in various conditions reveal also a wide distribution of activation energies at temperatures below $\mathrm{T}_{\mathrm{M}}$ [7]. It can be seen in figure 4 . as the low temperature tail of spectra for high voltages.

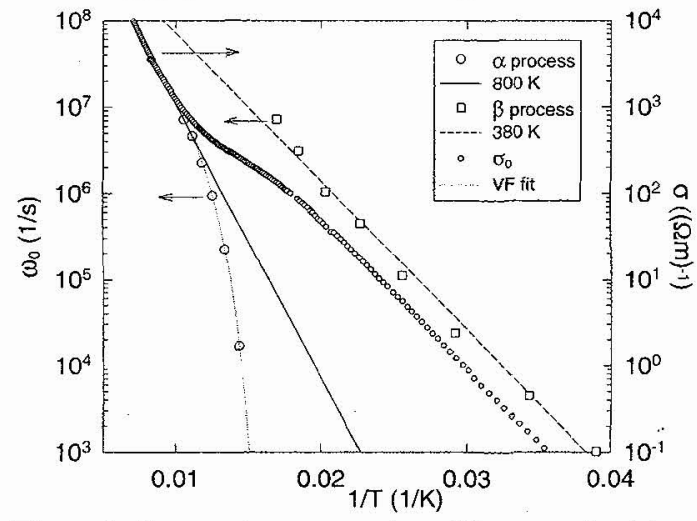

Figure 3. Comparative presentation of linear conductivity and characteristic frequencies of two processes. Full line represents the dependence expected for $\alpha$ process from measurements in higher temperature and frequency range [8] and theory [9]. The pointed line is Vogel-Fulcher fit.

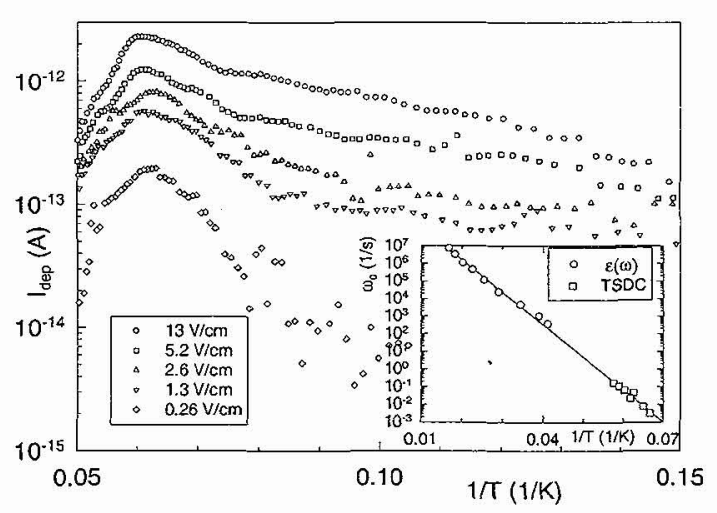

Figure 4. TSDC spectra for different polarization voltages and the same heating rate $h$ of $7 \mathrm{~K} / \mathrm{min}$. The inset shows the effective frequency versus position of maximum for $h$ between 0.2 and $20 \mathrm{~K} / \mathrm{min}$ together with characteristic frequencies of $\beta$ process.

These two processes and their properties are a direct proof of existence of different ground states of $\mathrm{CDW}$. The first one is dominated by collective pinning of elastic $\mathrm{CDW}$ in random potential. Below $100 \mathrm{~K}$ the condensation of free carriers leads to the Coulomb hardening of CDW and freezing of collective excitations below $50 \mathrm{~K}$. Only local degrees of freedom, like topological defects around strong impurities, remain at lower temperatures and the properties of a new ground state CDW are due to their dynamics.

\section{References}

[1] T. Takoshima et al., Solid State Commun. 35, (1980) 911

[2] F.Ya Nad', Pis'ma Zh.Eksp.Teor.Fiz. 58 (1993) 107, S.V. Zaitsev-Zotov, Phys.Rev.Lett. 71 (1993) 605

[3] J. Cava et al., Phys. Rev. B 31 (1985) 8325, F. Nad and P. Monceau, Phys, Rev. B 51, 2052 (1995)

[4] J. van Turnhout, in Electrets, Topics in Applied Physics 33, Springer-Verlag, Berlin, pp. 81-201

[5] K. Biljakovic et al., Physica B 244 (1998) 167

[6] D. Staresinic et al., proceedings of ICSM 98, to be published in Synth. Metals (1999)

[7] J. R. Tucker et al., Phys. Rev. B 34 (1986) 9038

[8] P. Littlewood, Phys. Rev. B 36 (1987) 3108 